\title{
Preoperative Low-Molecular-Weight Heparin Prophylaxis Associated with Increased Heparin Resistance Frequency in On-Pump Coronary Artery Bypass Graft Surgery
}

\author{
Onur Saydam (D), ${ }^{1}$ Mehmet Atay, ${ }^{2}$ Deniz Serefli, ${ }^{1}$ Suat Doganci, ${ }^{3}$ Ulas Kumabasar, ${ }^{4}$ \\ Mustafa Yılmaz, ${ }^{4}$ Riza Dogan, ${ }^{4}$ and Metin Demircin ${ }^{4}$ \\ ${ }^{1}$ Tepecik Training and Research Hospital, Department of Cardiovascular Surgery, Izmir 35180, Turkey \\ ${ }^{2}$ Bakirkoy Dr. Sadi Konuk Training and Research Hospital, Department of Cardiovascular Surgery, Istanbul 34147, Turkey \\ ${ }^{3}$ Gulhane Training and Research Hospital, Department of Cardiovascular Surgery, Ankara 06010, Turkey \\ ${ }^{4}$ Hacettepe University Faculty of Medicine, Department of Cardiovascular Surgery, Ankara 06230, Turkey
}

Correspondence should be addressed to Onur Saydam; onursaydam@hotmail.com

Received 29 August 2018; Revised 9 December 2018; Accepted 18 March 2019; Published 16 April 2019

Guest Editor: Frederik Trinkmann

Copyright $\odot 2019$ Onur Saydam et al. This is an open access article distributed under the Creative Commons Attribution License, which permits unrestricted use, distribution, and reproduction in any medium, provided the original work is properly cited.

Background. Unfractionated heparin (UFH) and low-molecular-weight heparin (LMWH) are being used for preoperative management of critical coronary artery disease. However, preoperative UFH therapy may cause a reduction in antithrombin concentrations, leading to various degrees of heparin resistance (HR). The main purpose of this study is to investigate the effects of preoperative LMWH on HR during cardiopulmonary bypass (CPB). Methods. Data were retrospectively reviewed from adult patients that underwent on-pump coronary artery bypass graft (CABG) surgery. Four hundred fifty-seven patients underwent CABG, and 139 of them, who had isolated on-pump CABG, were included in the study. The heparin sensitivity index was calculated if activated clotting time levels were discovered below 400 seconds. Values less than 1.3 were accepted as HR. Results. Of 139 patients who underwent on-pump CABG, preoperative LMWH was administered in 59 patients (56.8\%). Intraoperative HR occurred in 29 patients (20.9\%). Patients who received preoperative LMWH had an increased risk of developing HR compared with patients who did not receive LMWH (odds ratio 4.8 and 95\% confidence interval 1.7-13.5). CPB duration and aortic clamp duration were significantly longer in patients who developed intraoperative HR when compared to those in patients who did not develop HR. Conclusion. Preoperative treatment with LMWH may cause intraoperative HR. Corrective and preventive arrangements with close follow-up should be performed in this group of patients.

\section{Introduction}

Unfractionated heparin (UFH) is still the most reliable medication to prevent thrombosis and its catastrophic consequences during cardiopulmonary bypass (CPB). UFH indirectly inhibits coagulation by binding antithrombin (AT) [1]. UFH and AT complex bind thrombin, and UFH diverges from the complex. Afterward, thrombin-AT complex is consumed and eliminated by the reticuloendothelial system which leads to a decrease in AT levels. Preoperative UFH therapy may cause a reduction in AT concentrations, leading to various degrees of heparin resistance (HR) with this mechanism. CPB-associated $\mathrm{HR}$ is defined as the need for a higher dose of UFH than the standard dose to induce sufficient active coagulation time for CPB $[1,2]$. HR is reported in up to $22 \%$ of patients undergoing CPB $[2,3]$.

AT deficiency is the primary mechanism of $\mathrm{HR}$ and can be either congenital or acquired. Acquired AT deficiency may associate with advanced liver disease, renal dysfunction, such as nephrotic syndrome, and malnutrition. Also, an upregulated hemostatic system such as disseminated intravascular coagulation, deep vein thrombosis, and endocarditis may lead to AT deficiency. However, one of the most 
common causes for acquired AT deficiency is a preoperative $\mathrm{UFH}$ treatment [4]. Although $\mathrm{HR}$ is more common in patients who receive UFH preoperatively, it may also be seen in patients treated with low-molecular-weight heparin (LMWH) [5, 6]. LMWH is frequently being used for preoperative prophylaxis of the coronary artery disease, especially in patients with critical stenosis [5-8]. Even though LMWH has fewer binding sites for AT, both LMWH and $\mathrm{UFH}$ require AT for their anticoagulant effect. As a result, UFH inactivates factor Xa and factor IIa equivalently, while LMWH mostly inactivates factor Xa [9-11].

The main purpose of this study is to investigate the effects of preoperative LMWH on $\mathrm{HR}$ in patients who underwent on-pump CABG surgery.

\section{Methods}

Data of adult patients that underwent on-pump CABG surgery in a single center from January 2012 to January 2014 were retrospectively collected by a file scanning method. The Institutional Ethics Committee of Hacettepe University approved the study protocol (GO 14/171-15). Among 457 patients who underwent CABG surgery, 139 patients who had isolated on-pump CABG surgery were included in the study. The rest of the patients, who had redo, off-pump, emergency CABG, or combined CABG and valve surgery, were excluded from the study.

To eliminate other factors that cause acquired AT deficiency in patients with malnutrition and liver or renal dysfunction including nephrotic syndrome, were excluded. Patients previously diagnosed with AT deficiency and personal or family history of a thromboembolic event were also excluded. We excluded 28 patients from the study with a personal or family history of a thromboembolic event.

2.1. Standard Preoperative and Intraoperative Anticoagulation Protocol. LMWH was administered preoperatively to patients with critical coronary artery disease (CAD) (significant left main CAD or left main equivalent CAD). Enoxaparin was used as an LMWH in all patients with a subcutaneous dosage of $0.01 \mathrm{cc} / \mathrm{kg}$ body weight. LMWH was administered twice daily for at least three preoperative days and was stopped 24 hours before surgery. After induction and intubation, baseline ACT was measured by Hemochron $801^{\circledR}$ device (Technidyne Corp., Edison, New Jersey, USA). Before cannulation, UFH with a dosage of $300 \mathrm{U} / \mathrm{kg}$ was administered through the right atrium and the ACT level was measured 5 minutes after UFH injection. CPB was commenced, when ACT level was over 400 seconds. When ACT level was below 400 seconds, additional UFH (150 U/kg) was applied. ACT was monitored every 20 minutes during CPB. After discontinuation of CPB, $1 \mathrm{mg}$ of protamine was used to neutralize each $100 \mathrm{IU}$ of heparin, and the ACT level was measured 5 minutes after the completion of protamine.

2.2. Heparin Sensitivity Index Calculation. After a retrospective data revision, for patients with an ACT level below
400 seconds after the first standard heparin dose, Heparin Sensitivity Index (HSI) was calculated by the following formula: ACT after UFH - baseline ACT/overall loading dose of heparin (IU/kg) [12]. Values less than 1.3 were accepted as HR.

2.3. Patient Population. Patients were stratified into 4 groups according to the medical regimen and the presence of HR. Group 1 consisted of patients who did not receive LMWH before surgery and did not experience intraoperative $\mathrm{HR}$ (LMWH- / HR-). Group 2 consisted of patients that did not receive LMWH before surgery, but experienced intraoperative HR (LMWH-/HR+). Group 3 consisted of patients who received subcutaneous LMWH preoperatively, but did not experience intraoperative HR (LMWH+ / HR-). Group 4 consisted of patients who received subcutaneous LMWH preoperatively and experienced intraoperative HR $(\mathrm{LMWH}+/ \mathrm{HR}+)$ (Figure 1).

2.4. Study Parameters. Demographic data including age, gender, and weight, preoperative data including usage of acetylsalicylic acid (ASA) and clopidogrel, blood types, platelet counts, and perioperative baseline ACT levels, post$\mathrm{UFH}$ and post-protamine sulfate ACT levels, CPB time, aortic clamp time, unit of fresh frozen plasma (FFP), and thrombocyte given were collected. Length of stay in intensive care unit and length of hospital stay were also evaluated.

2.5. Statistics. Sample size was calculated, and with this sample size, there is above $85 \%$ likelihood that this study will yield statistically significant results. To test the normality of distribution, the Shapiro-Wilk test was used. Continuous, normally distributed data are presented as mean and standard deviation. Nonnormally distributed interval data are also presented with the lowest and highest scores. Categorical data are presented as frequencies and percentages. To test differences for preoperative serum platelet levels, the Kruskal-Wallis test was used. Pair-wise comparisons of groups were performed with the Conover-Dunn test. Pearson's correlation test was performed to show a relation between lower platelet counts in patients who developed intraoperative HR. Logistic regression analysis was used to understand independent factors which affect HR. G* Power 3 software was used to calculate achieved power for significant differences. A $P$ value less than 0.05 was considered statistically significant, and a $\beta$ type error was mentioned by $<0.20$ to represent achieved power.

\section{Results}

One hundred thirty-nine patients were evaluated. Patient demographics, clinical characteristics, and operative data are shown in Table 1. Group 1 (LMWH-/HR-) consisted of 55 patients, Group 2 (LMWH-/HR+) consisted of 5 patients, 


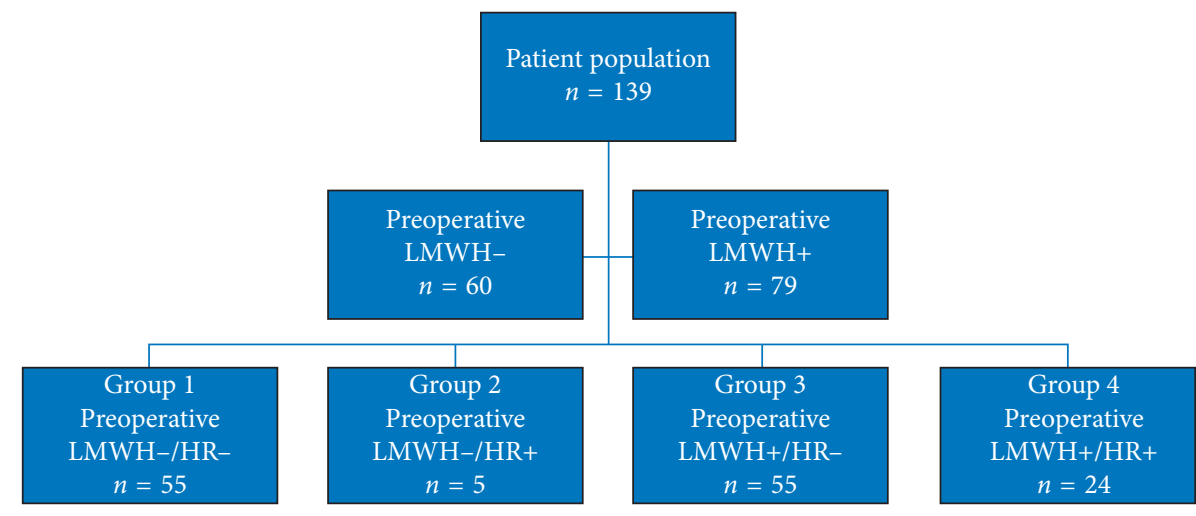

FIgURE 1: Patient population diagram. LMWH, low-molecular-weight heparin; HR, heparin resistance.

Table 1: Patient demographics, clinical characteristics, and operative data.

\begin{tabular}{|c|c|c|c|c|c|}
\hline & \multicolumn{2}{|c|}{$\operatorname{HR}-(n=110)$} & \multicolumn{2}{|c|}{$\mathrm{HR}+(n=29)$} & \multirow[b]{2}{*}{$P$ value } \\
\hline & $\begin{array}{l}\text { Group } 1 \text { LMWH- } \\
(n=55)\end{array}$ & $\begin{array}{c}\text { Group } 3 \text { LMWH+ } \\
(n=55)\end{array}$ & $\begin{array}{l}\text { Group } 2 \text { LMWH- } \\
\qquad(n=5)\end{array}$ & $\begin{array}{l}\text { Group } 4 \text { LMWH+ } \\
(n=24)\end{array}$ & \\
\hline Age (y) & $66 \pm 10$ & $63 \pm 9$ & $63 \pm 11$ & $65 \pm 11$ & 0.463 \\
\hline Gender (F) $(n)$ & 22 & 20 & 2 & 6 & 0.658 \\
\hline Weight (kg) & $77 \pm 12$ & $80 \pm 12$ & $77 \pm 7$ & $80 \pm 16$ & 0.598 \\
\hline $\begin{array}{l}\text { Preoperative platelet count } \\
(\times 1000 \mu \mathrm{L})\end{array}$ & $249 \pm 74$ & $275 \pm 85$ & $168 \pm 70$ & $197 \pm 75$ & $<0.001^{*}$ \\
\hline \multicolumn{6}{|l|}{ Intraoperative patients' data } \\
\hline $\mathrm{CPB}$ time (min) & \multicolumn{2}{|c|}{$67.8 \pm 26.0$} & \multicolumn{2}{|c|}{$94.2 \pm 55.9$} & $0.033^{\alpha}$ \\
\hline Aortic clamp time (min) & \multicolumn{2}{|c|}{$45.5 \pm 17.9$} & \multicolumn{2}{|c|}{$51.52 \pm 18.7$} & $0.022^{\alpha}$ \\
\hline Basal ACT (sec) & $126 \pm 16$ & $125 \pm 15$ & $119 \pm 18$ & $122 \pm 15$ & 0.654 \\
\hline Postheparin ACT (sec) & \multicolumn{2}{|c|}{$633.58 \pm 205.1$} & \multicolumn{2}{|c|}{$450.69 \pm 133.2$} & $0.001^{\alpha}$ \\
\hline Postprotamine ACT (sec) & $133 \pm 22$ & $154 \pm 7$ & $128 \pm 19$ & $133 \pm 25$ & $0.034+$ \\
\hline Intraoperative FFP used (units) & $2.1 \pm 0.9$ & $3.8 \pm 1.8$ & $2.1 \pm 0.9$ & $4.5 \pm 1.8$ & $<0.001^{\mathrm{x}}$ \\
\hline $\begin{array}{l}\text { Intraoperative thrombocyte used } \\
\text { (units) }\end{array}$ & $0.3 \pm 1.3$ & $1.0 \pm 2.2$ & $0.3 \pm 1.5$ & $2.2 \pm 3.1$ & $0.001^{\mathrm{x}}$ \\
\hline Reexploration $(n)$ & 0 & 0 & 0 & 2 & 0.980 \\
\hline Mortality $(n)$ & 1 & 0 & 0 & 4 & $0.013^{\mathrm{y}}$ \\
\hline
\end{tabular}

Data are presented as mean \pm SD. LMWH, low-molecular-weight heparin; HR, heparin resistance; $n$, number; y, years; F, female; kg, kilograms; CPB, cardiopulmonary bypass; min, minutes; ACT, active coagulation time; sec, seconds; FFP, fresh frozen plasma. * Significant difference was between Groups 2 and 3. ${ }^{\alpha}$ Significant difference was between groups HR+ and HR-. ${ }^{\mathrm{x}}$ Significant difference was between Groups 1 and 4 and Groups 2 and $4 .{ }^{\mathrm{y}}$ Significant difference was between Groups 2 and 4 and Groups 3 and 4.

Group 3 (LMWH+/HR-) consisted of 55 patients, and Group 4 (LMWH+/HR+) consisted of 24 patients (Figure 1). There were no significant differences among the groups regarding age, gender, weight, preoperative usage of ASA and clopidogrel, blood type, length of stay in intensive care unit, and length of hospital stay.

Seventy-nine $(56.8 \%)$ patients received LMWH preoperatively, and 29 (20.9\%) patients experienced intraoperative HR. We found LMWH as an independent factor that affects $\mathrm{HR}$ and the rate of heparin resistance is increased 4.8 times in the patients who used LMWH before surgery (95\% confidence interval 1.7-13.5, $P$ value 0.003 ). There were significant differences for preoperative serum platelet levels. Those differences in platelet counts were between Group 2 $(\mathrm{LMWH}-/ \mathrm{HR}+)$ and Group 3 (LMWH+/HR-) $(168 \pm 70(\times 1000 \mu \mathrm{L})$ vs $275 \pm 85(\times 1000 \mu \mathrm{L})$, respectively, $p=0.036)$ and between Group 3 (LMWH+/HR-) and Group $4 \quad(\mathrm{LMWH}+/ \mathrm{HR}+) \quad(275 \pm 85(\times 1000 \mu \mathrm{L}) \quad$ vs $197 \pm 75(\times 1000 \mu \mathrm{L})$, respectively $p=0.001)$. There was a correlation between lower platelet counts with intraoperative HR $(p<0.001)$ (coefficient correlation -0.342). Basal levels of ACT were similar among the groups. Mean ACT levels following 5 minutes after the first UFH administration showed significant differences between the groups HR+ and HR- $(450.69 \pm 133.2$ vs $633.58 \pm 205.1$, respectively, $p<0.001)$. ACT levels 5 minutes after protamine sulfate administration also showed a significant difference between groups with and without preoperative LMWH therapy $(p=0.034)$. ACT levels 5 minutes after protamine sulfate administration showed significant differences between Group 2 (LMWH-/ HR+) and Group 4 $(\mathrm{LMWH}+/ \mathrm{HR}+) \quad(154 \pm 7$ vs $133 \pm 25$, respectively, $p=0.032)$ and Group 2 (LMWH-/HR+) and Group 3 $(\mathrm{LMWH}+/ \mathrm{HR}-) \quad(154 \pm 7$ vs $128 \pm 19$, respectively, $p=0.024)$. Only $\mathrm{HR}+$ patients received intraoperative additional doses of UFH $(150 \mathrm{U} / \mathrm{kg})$, and there was no significant difference between Group 2 (LMWH-/HR+) and Group 4 (LMWH+/HR+). 
Significant differences were found between the $\mathrm{HR}+$ and HR- patients in terms of CPB duration and aortic clamp duration $(94.2 \pm 55.9 \mathrm{~min}$ vs $67.8 \pm 26.0 \mathrm{~min}, p=0.033$ and $51.52 \pm 18.7 \mathrm{~min}$ vs $45.5 \pm 17.9 \mathrm{~min}, p=0.022)$. We performed a power analysis to exclude underpowered outcomes. Variables such as LMWH, CPB time, and aortic clamp time achieved power more than $85 \%$. Only 2 patients needed mediastinal re-exploration due to excessive bleeding, and both patients were in Group 4 (LMWH+/HR+).

Compared to all other groups, mortality was significantly higher in Group $4(n=4)(\mathrm{LMWH}+/ \mathrm{HR}+)(p=0.013)$.

\section{Discussion}

This current article shows the relation between preoperative usage of LMWH and HR. The definition of the causes and effects of HR are still controversial in cardiac surgery practice. There are very few studies examining the effects of preoperative LMWH therapy on HR during cardiac surgery $[5,13]$. In this study, we specifically compared adult patients undergoing on-pump CABG surgery who received preoperative LMWH with a control group who did not receive LMWH before surgery. The overall incidence of intraoperative HR was $20.9 \%(n=29)$, which was similar to that of other studies [2,3]. The incidence of intraoperative HR was observed significantly higher in the group with preoperative LMWH treatment. These results were similar to the previously published data of preoperative UFH treatment $[14,15]$. LMWH and UFH both exert their effects through the same AT pathway. UFH leads to equivalent inactivation of factor X and factor IIa, while LMWH mostly leads to inactivation of factor X. Since both drugs act over AT pathway for anticoagulant effect, this may be the explanation of similar results $[9,10]$. Most recent investigations provide evidence that there is a significant positive correlation between preoperative platelet count and intraoperative HR development $[12,16,17]$. However, there are contradictory results in the literature. Brinks et al. [18] demonstrated a relation between preoperative lower platelet counts in patients who developed intraoperative HR. Our results were also parallel to their findings. Although preoperative serum platelet levels were in the normal range in all groups, platelet levels were significantly lower in $\mathrm{HR}+$ groups (Group 2 and Group 4). In contrast to our study, Brinks et al. [18] did not demonstrate any difference in platelet counts between groups with and without LMWH pretreatment and concluded that this was most likely due to the inadequate definition of HR. As mentioned before, the ACT response to UFH is complex and affected by different factors. Although AT pathway is the main mechanism for the anticoagulant effect of UFH, there are other factors, such as plasma protein binding, leukocyte, lactoferrin, and activated platelet count [3].

Dietrich and et al. [14] demonstrated that patients with intraoperative HR showed prolonged CPB and aortic clamp times. Results of the current study were also similar to these findings. However, there are also studies that show no effect of $\mathrm{CPB}$ and aortic clamp times on $\operatorname{HR}[7,18]$. These results are proof of an ongoing discussion.
ACT levels 5 minutes after the administration of protamine sulfate was also measured in this study. Kanbak et al. [19] found no significant difference between HR+ and HRgroups in terms of ACT levels after protamine. In this study, ACT levels after protamine in LMWH pretreated patients were higher, but the comparison was irrespective of HR presence. Unlike UFH, LMWH agents have longer half-lives [11]. Cavusoglu et al. [20] showed in their study that LMWH agents might have an effect on ACT levels.

There are intraoperative treatment strategies for $\mathrm{HR}$, such as fresh frozen plasma (FFP) or AT administration. Because of its easy accessibility and lower prices, FFP is the most common therapy in current practice. In this study, it was found that Group $4(\mathrm{LMWH}+/ \mathrm{HR}+)$ had received significantly higher amounts of intraoperative FFP $(p<0.001)$. Although this increased FFP requirement might be explained by the defect of an intraoperative bleeding profile of Group 4, there was no significant difference between the groups in terms of mediastinal re-exploration due to bleeding complications. However, there are many studies showing higher rates of mediastinal re-exploration in LMWH pretreated patients [7]. This might show the effectiveness of adequate intraoperative FFP therapy to prevent bleeding complications. Our mortality rate was 3.6\% $(n=5)$. Four out of five patients that died were in Group 4, and mortality rate was found significantly higher in Group 4 $(\mathrm{HR}+/ \mathrm{LMWH}+)$ than other groups. Ranucci et al. [3] also showed significantly higher rates of mortality in HR groups, even though there was no difference in terms of patient demographics. However, in our study, LMWH pretreated patients had more critical coronary artery diseases, which can be one of the reasons to explain higher rates of mortality.

Besides the findings of this study, there are some limitations. This study was conducted in a single institution with a limited sample size. Our study is a retrospective design study, laboratory tests for congenital or acquired AT deficiencies are not in our routine preoperative screening, and we could not identify the undiagnosed AT deficiencies. Relatively small sample size might have precluded us to detect minor differences between the groups. The preoperative administration of enoxaparin was not controlled, and although there were no significant differences between the groups, effects of preoperative usage of ASA and clopidogrel on HR were not investigated. Progression of HR is strongly related to the dose of UFH to achieve a specific target ACT. Because of this strong relationship, our results may be estimated to higher target ACTs, and this might have affected our results. We found the longer duration of ICU and hospital stay in $\mathrm{HR}+$ groups. However, further prospective trials with homogenous groups are needed for the explanation of this relation between $\mathrm{HR}$ and postoperative outcome.

In conclusion, preoperative LMWH treatment may cause HR in patients undergoing on-pump CABG surgery. In general, practice to overcome $\mathrm{HR}$ during on-pump cardiac surgery higher amounts of UFH is used. Inevitably, using higher amounts of UFH may have effects on early postoperative outcome such as bleeding. Therefore, detection of preoperative HR should be a warning for 
postoperative events. Corrective and preventive management with close follow-up should be performed in this group of patients. Further prospective clinical research studies in larger patient populations are necessary to encourage our results.

\section{Data Availability}

The datasets generated and/or analyzed during the current study are available from the corresponding author on reasonable request.

\section{Ethical Approval}

The Ethical Committee of Hacettepe University Hospital approved the protocol and granted us permission to access the patient's data. All procedures performed in this study involving human participants were in accordance with the ethical standards of the institutional and/or national research committee and with the 1964 Helsinki Declaration and its later amendments or comparable ethical standards.

\section{Disclosure}

The data included in this manuscript have been presented as a poster at the $12^{\text {th }}$ International Congress of Update in Cardiology and Cardiovascular Surgery on March 10-13, 2016, in Antalya, Turkey.

\section{Conflicts of Interest}

The authors declare that they have no conflicts of interest regarding the publication of this paper.

\section{References}

[1] Y.-J. Chuang, R. Swanson, S. M. Raja, and S. T. Olson, "Heparin enhances the specificity of antithrombin for thrombin and factor $\mathrm{Xa}$ independent of the reactive center loop sequence," Journal of Biological Chemistry, vol. 276, no. 18, pp. 14961-14971, 2001.

[2] M. S. Avidan, J. H. Levy, J. Scholz et al., "A phase III, doubleblind, placebo-controlled, multicenter study on the efficacy of recombinant human antithrombin in heparin-resistant patients scheduled to undergo cardiac surgery necessitating cardiopulmonary bypass," Anesthesiology, vol. 102, no. 2, pp. 276-284, 2005.

[3] M. Ranucci, G. Isgrò, A. Cazzaniga et al., "Different patterns of heparin resistance: therapeutic implications," Perfusion, vol. 17, no. 3, pp. 199-204, 2002.

[4] S. Bucur, J. Levy, G. Despotis, B. Spiess, and C. Hillyer, "Uses of antithrombin III concentrate in congenital and acquired deficiency states," Transfusion, vol. 38, no. 5, pp. 481-498, 1998.

[5] S. Bar-Yosef, H. B. Cozart, B. Phillips-Bute, J. P. Mathew, and H. P. Grocott, "Preoperative low molecular weight heparin reduces heparin responsiveness during cardiac surgery," Canadian Journal of Anesthesia/Journal canadien d'anesthésie, vol. 54, no. 2, pp. 107-113, 2007.

[6] H. Pleym, V. Videm, A. Wahba et al., "Heparin resistance and increased platelet activation in coronary surgery patients treated with enoxaparin preoperatively," European Journal of Cardio-Thoracic Surgery, vol. 29, no. 6, pp. 933-940, 2006.

[7] S. B. McDonald, M. Renna, E. L. Spitznagel et al., "Preoperative use of enoxaparin increases the risk of postoperative bleeding and re-exploration in cardiac surgery patients," Journal of Cardiothoracic and Vascular Anesthesia, vol. 19, no. 1, pp. 4-10, 2005.

[8] J. A. M. Anderson and E. L. Saenko, "Editorial I," British Journal of Anaesthesia, vol. 88, no. 4, pp. 467-469, 2002.

[9] J. I. Weitz, "Low-molecular-weight heparins," New England Journal of Medicine, vol. 337, no. 10, pp. 688-699, 1997.

[10] J. Hirsh and R. Raschke, "Heparin and low-molecular-weight heparin," Chest, vol. 126, no. 3, pp. 188S-203S, 2004.

[11] M. M. Samama and F. Michaut-Paterno, "Therapeutic indications of low molecular weight heparins," Archives des Maladies du Coeur et des Vaisseaux, vol. 84, pp. 1733-1743, 1991.

[12] A. Vuylsteke, R. J. Mills, A. E. Crosbie, T. I. Burns, and R. D. Latimer, "Increased pre-operative platelet counts are a possible predictor for reduced sensitivity to heparin," British Journal of Anaesthesia, vol. 85, no. 6, pp. 896-898, 2000.

[13] M. H. Staples, R. F. Dunton, K. J. Karlson, H. K. Leonardi, and R. L. Berger, "Heparin resistance after preoperative heparin therapy or intraaortic balloon pumping," The Annals of Thoracic Surgery, vol. 57, no. 5, pp. 1211-1216, 1994.

[14] W. Dietrich, M. Spannagl, W. Schramm, W. Vogt, A. Barankay, and J. A. Richter, "The influence of preoperative anticoagulation on heparin response during cardiopulmonary bypass," Journal of Thoracic and Cardiovascular Surgery, vol. 102, no. 4, pp. 505-514, 1991.

[15] T. Chan, N. C. Hwang, and C. H. Lim, "A statistical analysis of factors predisposing patients to heparin resistance," Perfusion, vol. 21, no. 2, pp. 99-103, 2006.

[16] K. Bagheri, A. Honarmand, M. Safavi, P. Kashefi, L. Sayadi, and L. Mohammadinia, "The evaluations of frequency distribution heparin resistance during coronary artery bypass graft," Advanced Biomedical Research, vol. 3, no. 1, p. 53, 2014.

[17] S. Garvin, D. Fitzgerald, J. D. Muehlschlegel et al., "Heparin dose response is independent of preoperative antithrombin activity in patients undergoing coronary artery bypass graft surgery using low heparin concentrations," Anesthesia \& Analgesia, vol. 111, pp. 856-861, 2010.

[18] H. Brinks, P. Weerwind, S. Bogdan, H. Verbruggen, and M. Brouwer, "Does heparin pretreatment affect the haemostatic system during and after cardiopulmonary bypass?," Perfusion, vol. 16, no. 1, pp. 3-12, 2001.

[19] M. Kanbak, B. Oc, M. A. Salman, T. Ocal, and M. Oc, "Peroperative effects of fresh frozen plasma and antithrombin III on heparin sensitivity and coagulation during nitroglycerine infusion in coronary artery bypass surgery," Blood Coagulation \& Fibrinolysis, vol. 22, no. 7, pp. 593-599, 2011.

[20] E. Cavusoglu, M. Lakhani, and J. D. Marmur, "The activated clotting time (ACT) can be used to monitor enoxaparin and dalteparin after intravenous administration," Journal of Invasive Cardiology, vol. 17, no. 8, pp. 416-421, 2005. 


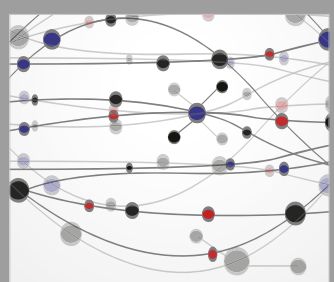

The Scientific World Journal
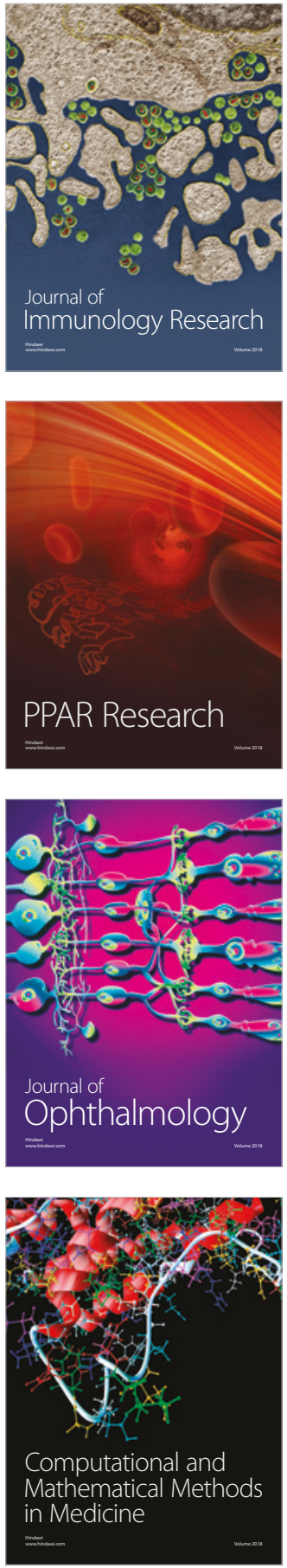

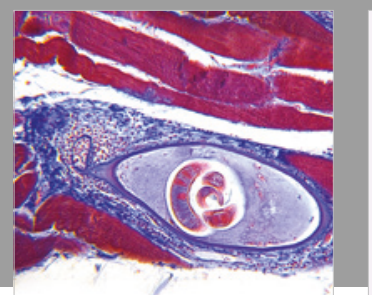

Gastroenterology Research and Practice

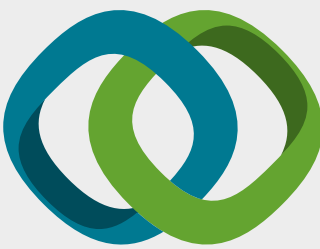

\section{Hindawi}

Submit your manuscripts at

www.hindawi.com
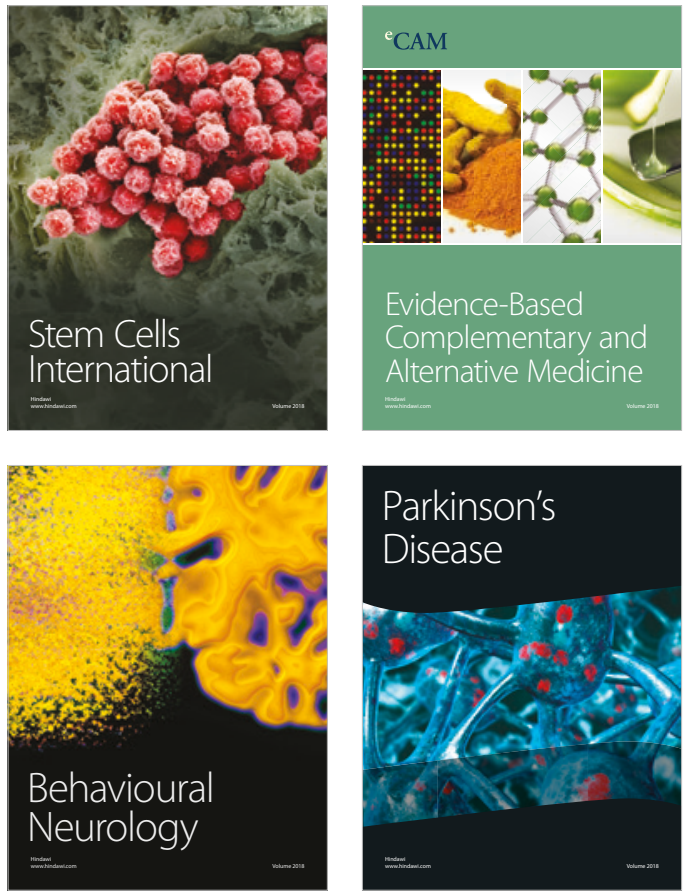

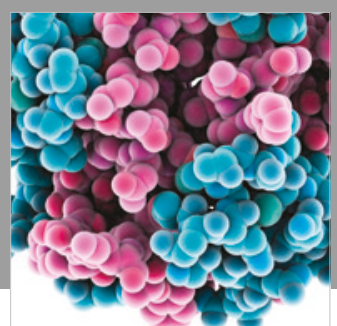

ournal of

Diabetes Research

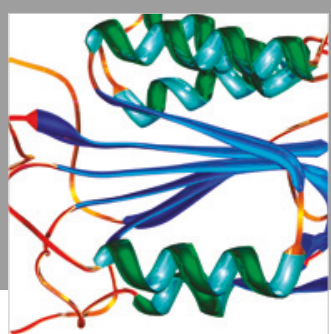

Disease Markers
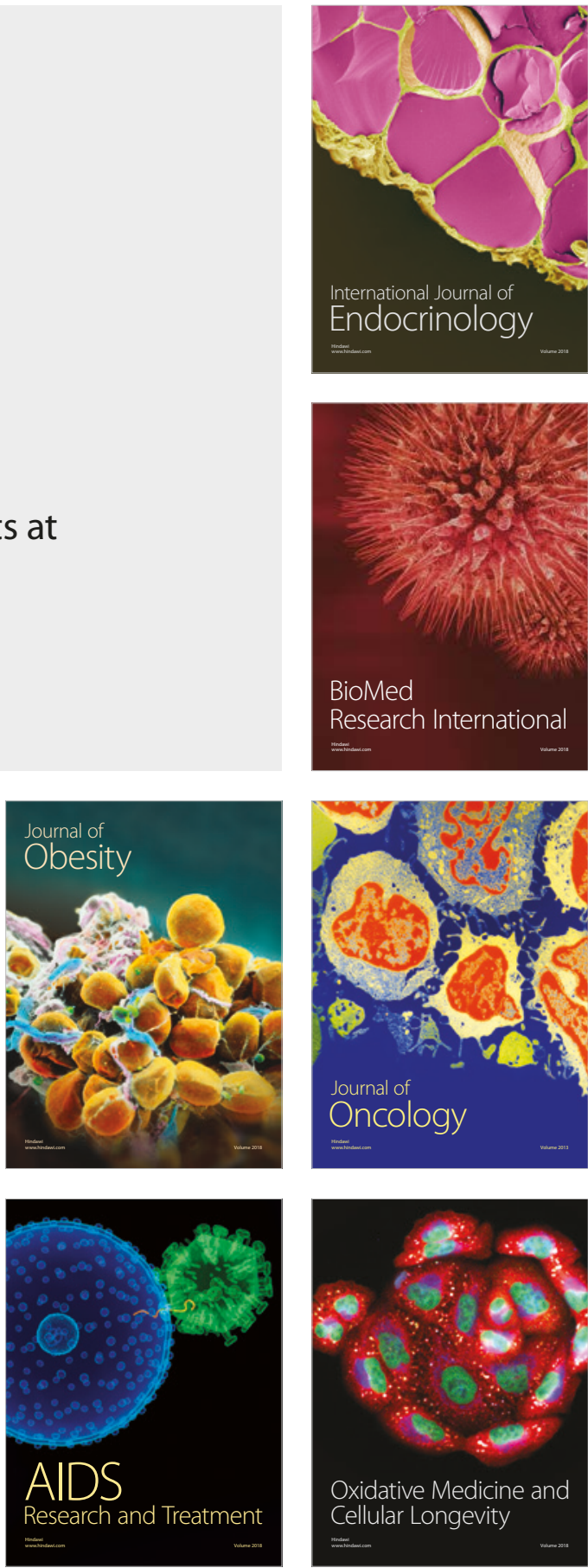